\title{
Optically tethered interfacing
}

IEEE Trans. Biomed. Circuits Syst. https://doi.

org/10.1109/TBCAS.2018.2876069 (2018)

Neural interfaces enable monitoring and recording of brain activity, and could provide valuable information to those working to develop treatments for individuals with sensorimotor impairments and neurological diseases. Long-term monitoring of neural activity in active animals remains challenging, as data and power transfer requires the use of tethered implants that are not practical on a day-to-day basis. To overcome this issue, researchers have started to focus on developing untethered systems that can transmit data and power via radio frequency (RF) or ultrasonic radiation. However, these systems are often bulky or inefficient.

Sunwoo Lee and colleagues at Cornell University have now developed a tetherless neural interface based on $180 \mathrm{~nm}$ complementary metal-oxidesemiconductor (CMOS) technology. Their approach, which they term a microscale opto-electronically transduced electrode (MOTE), uses an integrated AlGaAs diode that functions as both a photovoltaic cell and a light-emitting diode, enabling the transfer of power and data at comparatively deep penetration depths, and with minimal displacement of tissue. The device is $250 \mu \mathrm{m} \times 57 \mu \mathrm{m}$ in size, weighs $11 \mu \mathrm{g}$, and consumes $1 \mu \mathrm{W}$. It is able to measure low-noise neural signals, offering several significant benefits over current RF- and ultrasonic-based tetherless neural interfaces.

\section{Stuart Thomas}

Published online: 12 November 2018 https://doi.org/10.1038/s41928-018-0170-5 